\title{
A Novel Demand Transition Procedure for Outage Risk Reduction in Distribution Networks
}

\author{
Yunche Su, Junyong Liu, Youbo Liu \\ School of Electrical Information and Engineering \\ Sichuan University \\ Chengdu, China \\ suyc@stu.scu.edu.cn
}

\author{
Gareth A. Taylor \\ College of Engineering, Design and Physical Sciences \\ Brunel University London \\ London, UK \\ Gareth.Taylor@brunel.ac.uk
}

\begin{abstract}
In general the most highly developed urban distribution networks provide high levels of reliability. However, the majority urban distribution networks still face high levels of outage risk during peak-load periods, especially when the available back-up generation capacity is insufficient. In order to accurately assess the outage risk during such operational periods, four system outage risk evaluation indices are presented based on the impacts of the worst fault case in the medium voltage distribution feeder. As a consequence, in order to reduce the outage risk, a novel multiple time interval based demand transition optimization procedure is demonstrated in this paper. By considering the changes of load and backup generation output in the whole demand transition process, the continuity of restoration is guaranteed. The effectiveness of the proposed approach is validated by the case study.
\end{abstract}

Keywords-Demand transition, multiple time interval, outage risk, system reliability, distribution network

\section{INTRODUCTION}

The main purpose of distribution network is to provide customers with reliable and secure electricity. In order to enhance system reliability, many advanced technologies, e.g. meshed network upgrade, distribution automation, etc. are implemented all over the world [1]. Nowadays, most of the urban distribution networks can reach quite high levels of reliability as long as their backup generation capacity is sufficient. The average customer interruption duration is usually less than several minutes. However, in some countries, e.g. the US, China, etc., electricity load has a big difference in different seasons. Due to the extremely hot weather in summer, residential peak load consumption may be several times more than the average annual load because of widely use of air conditional. Even if such peak load period may only last a few hours, severe outage incidents may happen because the load demand cannot be transferred to other generations. Besides, the equipment in overloading conditions is more likely to cause a failure. In that case, these

This work was supported in part by National High Technology Research and Development Program of China (863 program) (2014AA051901). distribution networks are facing the situation of "high reliability, also high potential outage risks".

One of the main reasons why the phenomenon occurs is that the operational outage risk is usually not considered seriously in the distribution network planning process. Most of the reliability indices presented in the IEEE guide [2] only considered about the annual average reliability performance. As a result, although plenty of the distribution network planning researches [3-5] took reliability enhancement as one of the optimization objectives, the outage risk still cannot occupy an important position as the peak load period only has little influence on these reliability indices. The impact of some backup resources, e.g. tie line between feeders, distributed generation with energy storage system, etc. may also be underestimated because they won't make much progress to reliability improvement if the existing backup generation capacity is sufficient in most of the times [6].

On the distribution network operation level, an effective network reconfiguration and demand transfer strategy will also play an important role in reducing system outage risk. Many researches presented variety of reconfiguration optimization model and solving algorithms [7-8]. However, on one hand, most of the methods, e.g. loop cutting method, branch exchange method, etc., are more suitable for the network reconfiguration in normal conditions because the demand transfer in fault conditions needs rapid action for electricity restoration [9]. On the other hand, most of the existing researches only considered demand transfer strategy in a single time interval while assuming load and generation output are constant during the whole transfer process [10-11]. However, demand transfer in fault conditions is a dynamic procedure, the strategies in different time intervals may influence each other. Some strategies may be infeasible even if it is optimized at the fault beginning moment because the load and generation output would be changing afterwards.

In order to solve the aforementioned problems. Two main contributions are made in this paper. First, in order to emphasize the system risk in peak load period, four outage risk evaluation indices for medium-voltage distribution network are presented based on the worst fault condition. Second, a multiple time intervals based demand transfer 
model is established for the reduction of system outage risk. The model set the minimization of outage rick index as objective and is solved by heuristic method. The effectiveness of the proposed approach is validated in the IEEE RBTS-Bus 3 distribution network.

\section{OUTAGE RISK EVALUATION INDICES}

In medium-voltage distribution networks, high levels of reliability performance depend largely on fast and effective operation of fault isolating and demand transferring. However, during peak load periods, if the load ratio in most of feeders is all in high levels, there will be great challenges in demand transfer process. Fig.1 shows a typical peak load day in Kansas, US. The picture illustrates that on one hand, the amount of the load demand that need to be transferred in fault conditions is large (red line in Fig.1). On the other hand, as the adjacent feeders also have heavy loads, the transfer capacity provided by tie lines is lower than normal (grey line in Fig.1). If a fault happens in such periods, customers will probably have a long interruption duration as the transfer capacity is insufficient (shaded area in Fig.1). In other words, the distribution network is facing severe system outage risk.

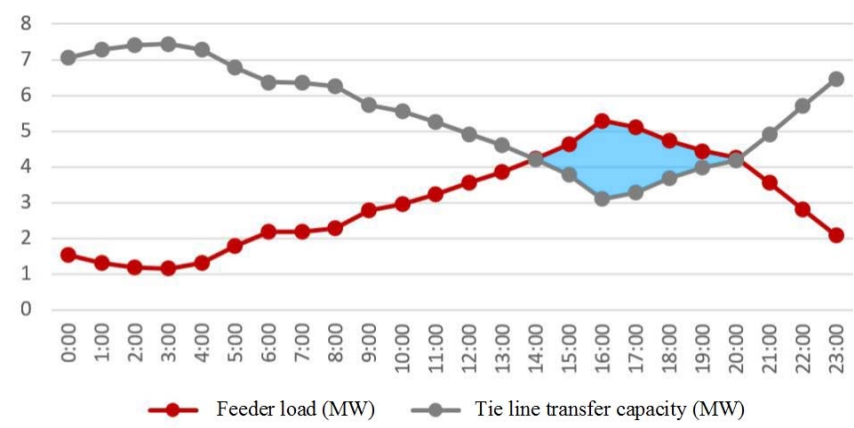

Fig.1 Load demand and tie line transfer capacity in a typical peak load day

The evaluation and reduction of system outage risk is helpful to ensure customers' electricity quality. It is useful for both distribution network planning stuff and distribution network operator. However, most of the existing reliability evaluation indices, e.g. system average interruption frequency index (SAIFI), system average interruption duration index (SAIDI), customer interruption (CI), customer minute lost (CML), energy not supply (ENS), can only assess the general reliability performance of distribution network. Although they have been widely used in distribution network planning process, all of them cannot evaluate the operational outage risk properly. Because the peak load period only account for a very short time in the whole year, even a severe outage event would have little influence on such indices either by using analytical or simulation methods.

In order to accurately evaluate the system outage risk, four outage risk indices are proposed in this paper. The evaluation contents of the indices are similar to those of reliability indices. Both of them assess the consequences of system faults. However, the risk evaluation indices are specifically focusing on the impact of the worst fault case of a mediumvoltage feeder. Typically, the fault case is under the following conditions.
- The fault happens at the beginning of the feeder, which means all of the load demand needs to be transferred to backup generations.

- The fault needs to be repairing for the whole time period when the transfer capacity is insufficient, which means load demand needs to be transferred during all of these time intervals (from 14:00 to 20:00 in Fig.1).

\section{A. Risk of customer minutes lost (RCMI)}

RCMI evaluates the average customer interruption minutes caused by the worst fault case. It is expressed as

$$
R C M I=\sum_{i \in T_{F}} \sum_{j \in C_{N}} T_{I} N_{j} \alpha_{i, j} / \sum_{j \in C_{N}} N_{j}
$$

where $T_{F}$ is the set of time intervals during the fault, $C_{N}$ is the set of customer nodes, $T_{I}$ is the length of each time interval, $N_{j}$ is the number of customers at node $j, \alpha_{i, j}$ is binary variable representing interruption situations. $\alpha_{i, j}$ equals 1 when the node $j$ is interrupted at time interval $i$, equals 0 when it is not.

\section{B. Risk of energy not supply (RENS)}

RENS evaluates the total energy lost caused by the worst fault case. Similar to RCMI, it is expressed as

$$
R E N S=\sum_{i \in T_{F}} \sum_{j \in C_{N}} T_{I} P_{i, j}^{L} \alpha_{i, j}
$$

where $P_{i, j}^{L}$ is the power of customers at node $j$.

\section{Risk of short time customer interruption (RSCI)}

RSCI evaluates the total number of short time interruption customers caused by the worst fault case. Customers are interrupted for a short time usually because of some necessary fault isolating process including operation of reclosure, sectional switch etc. Customers can be transferred to other generation after such processes so the interruption typically only last several minutes.

\section{Risk of long time customer interruption (RLCI)}

RSCI evaluates the total number of long time interruption customers caused by the worst fault case. Compared to short time interruption, long time interruption occurs because of the insufficient transfer capacity. The outage usually lasts several hours because the customers have to wait for the fault repairing process.

\section{TRANSITION OPTIMIZATION STRATEGY}

\section{A. Problem formulation}

In order to reduce system outage risk in the peak load period, it is significant to make the best use of all the back-up generations and develop effective demand transition strategy. The process is mathematically an optimization problem, which is formulated as

$$
\begin{cases}\min & f\left(x_{t}^{G}, x_{t}^{S}\right) \\ \text { s.t. } & h\left(x_{t}^{G}, x_{t}^{S}\right)=0 \\ & g\left(x_{t}^{G}, x_{t}^{S}\right) \leq 0\end{cases}
$$

The objective function $f(x)$ varies from different practical needs of electrical company. Typically, it is one of the reliability indices or the combination of them. Optimization variables consist of two parts. $x_{r}^{G}$ represents the output 
schedule of the back-up generations. Distributed generation (DG) and energy storage system (ESS) are considered in this paper. $x_{r}^{S}$ represents the switch operation in each time interval. The equality constraint $h(x)$ consists of power flow equation and maintenance of radial network structure. Line transmission capacity limitations and voltage drop limitations are included in the inequality constraint $g(x)$.

The key difficulties of solving this mix integer nonlinear optimization problem are listed follow.

- With the integrated multiple back up generation, there exists combinatorial explosion phenomenon for large scale scheduling candidates.

- Demand transfer is a dynamic process. Transfer strategy should consider the changes of load and generation during the multiple transfer time intervals.

- Transfer strategy needs to be optimized online right after fault happens. The efficiency of the solving approach must be guaranteed.

Considering the biggest difference between different load transfer strategies is the transfer route of each back-up generation, some transfer principles are set in advance in order to simplify the solving process. The principles are on the basis of practical experience of DNO.

- While a load node can be transferred to multiple backup generations, priority is given to more stable generations. Specifically, tie line is prior to DG with ESS, DG with ESS is prior to the ESS alone.

- While a backup generation can provide emergency electricity to multiple load node, the choice of the supply node is determined by the optimization objective. For example, if the objective is minimize RENS, the node with the largest load will be chosen.

- Each customer can only be interrupted once during the transfer process. That means once the electricity is restored to a customer, the continuously restoration need to be provided until the fault is repaired.

It is notable that the load node here represents an equivalent load area with sectional switches on its borders, which means the area cannot be separated any further in the demand transfer analysis. In addition, the DG without ESS is not set as backup generations because it is intermittent and uncontrollable. On the basis of the principles, the optimization process is simplified. Transfer route of each backup generation can be determined by heuristic algorithm.

Before the route search started, the initial capacity of each generation in each time interval is calculated by the formulas in Tab I.

TABLE I TRANSFER CAPACITY OF BACKUP GENERATIONS

\begin{tabular}{|c|c|}
\hline Backup Generation & Transfer Capacity in the Time Interval $\boldsymbol{i}$ \\
\hline Tie line & $P^{T L_{-} \text {max }}-P_{i}^{T F}$ \\
\hline PV with ESS & $\min \left(\mathrm{SOC}_{i} / T_{I}, P^{E_{-} \max }\right)+P_{i}^{P V}$ \\
\hline ESS & $\min \left(\mathrm{SOC}_{i} / T_{I}, P^{E_{-} \text {max }}\right)$ \\
\hline$P^{T L \_m a x}$ is the maximum transmission capacity of the tie
\end{tabular}

$P^{T L} P_{\text {max }}$ is the maximum transmission capacity of the tie line. $P_{i}^{T F}$ is the power of the other normal operation feeder connected to the tie line at time interval $i . S O C_{i}$ is the state of charge of the energy storage system at time interval $i . P^{E \_ \text {max }}$ is the maximum power output of the energy storage system. $P_{i}^{P V}$ is the power output of the PV at time interval $i$.

\section{B. Transfer route searching}

Assuming that the fault is happened in time $T_{1}$ and repaired in time $T_{2}$. The transfer route of each backup generation is determined by the following search procedure.

Step 1: Searching for every node that is connected to the backup generation.

Step 2: Choosing the next priori load node $j$ according to the demand transfer objective. If all nodes is transferred successfully, go to step 8. If no nodes can be transferred anymore, go to step 5 .

Step 3: For each time interval $i$ from $T_{2}$ to $T_{1}$, if the transfer capacity is larger than the load demand in node $j$, set the node transfer start time $T s_{j}$ (the initial value of $T s_{j}$ is zero) to $i$, else go to step 2.

Step 4: Determining whether $T s_{j}$ equals to $T_{1}$, if so, merge the node $j$ into the generation node, go to step 2, if not, go to step 2 directly.

Step 5: Searching for every node that is connected to load node $J$ as long as $T s_{J}$ is not equals to zero.

Step 6: Choosing the next priori load node according to the demand transfer objective. If no nodes can be transferred anymore, go to step 8.

Step 7: For each time interval $i$ from $T_{2}$ to $T s_{J}$, if the transfer capacity is larger than the load demand in node $j$, set the node transfer start time $T s_{j}$ to $i$, else go to step 6 .

Step 8: Output $T s_{j}$ of every load nodes.

To illustrate the procedure more clearly, a simple example of the transfer route search is presented. The basic parameter of the network is shown in Fig.2. The node 1 is the backup generation node while the other nodes are all load nodes. Assuming a fault happens in all of the four time intervals and the node transfer priority sequence is $2,4,3$, and 5 .
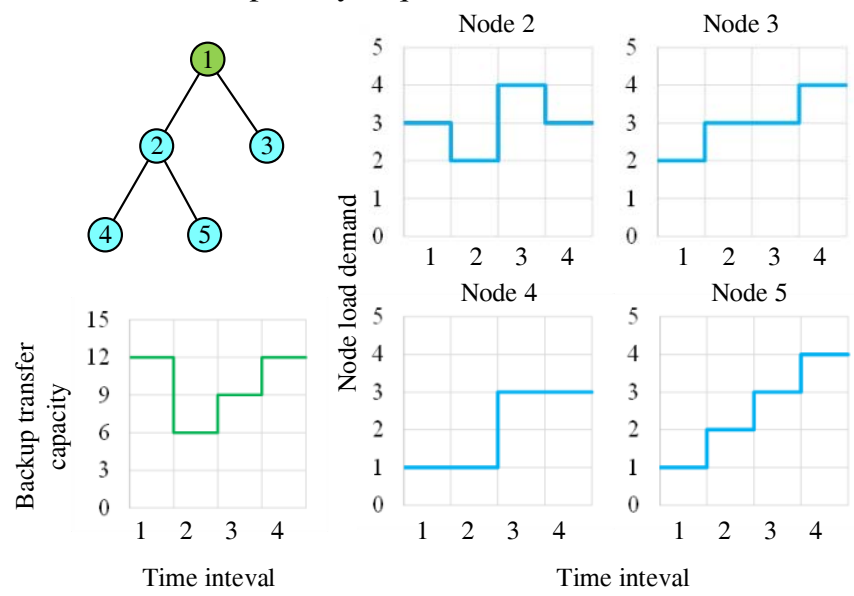

Fig.2 A simple example of transfer route searching

As the backup generation can provide energy to the node 2 and 4 in all the four time intervals, the two nodes will be restored at the beginning of time interval 1. After that, the rest of the backup generation capacity can only give electricity to load node 3 in the time interval 1,2 and 4. In order to avoid 
multiple times interruption (the first short interruption when the fault happens and the second in the time interval 3), the node 3 will be restored electricity only in time interval 4. As the remaining generation capacity in the time interval 4 is less than the load demand of node 5 , the node 5 will be interrupted during all of the four time intervals. As a result, $T s_{2}, T s_{3}, T s_{4}, T s_{5}$ equals $1,4,1$ and 0 respectively.

It is notable that the power flow and network structure is checked during every step 3 and step 7, if the voltage constraint or the radial network constraint is not satisfied, the strategy will be dropped and back to step 2 or step 6 to choose another option.

\section{Multiple generation merging}

During the transfer route searching process, one backup generation nodes may merge with another. In that condition, the demand transfer strategy needs to consider about the dispatch of different kinds of generations. In order to simplify the problem, generations are arranged in turns based on the priority principle. Specifically, in each time interval $i$, the route searching approach firstly determines whether the total generation capacity is enough for the load demand of node $j$. If so, the generations are dispatched by the following equations in turns.

$$
\begin{gathered}
C_{i}^{T R}= \begin{cases}0 & C_{i}^{T I} \leq P_{i, j}^{L} \\
C_{i}^{T I}-P_{i, j}^{L} & C_{i}^{T I}>P_{i, j}^{L}\end{cases} \\
C_{i}^{P R}= \begin{cases}0 & C_{i}^{T I}+C_{i}^{P I} \leq P_{i, j}^{L} \\
C_{i}^{P I}+C_{i}^{T I}-P_{i, j}^{L} & C_{i}^{T I}+C_{i}^{P I}>P_{i, j}^{L}\end{cases} \\
C_{i}^{E R}= \begin{cases}0 & C_{i}^{T I}+C_{i}^{P I}+C_{i}^{E I} \leq P_{i, j}^{L} \\
C_{i}^{P I}+C_{i}^{T I}+C_{i}^{E I}-P_{i, j}^{L} & C_{i}^{T I}+C_{i}^{P I}+C_{i}^{E I}>P_{i, j}^{L}\end{cases}
\end{gathered}
$$

where $P_{I}^{T I}, P_{I}^{P I}$ and $P_{i}^{E I}$ are the initial generation capacity of tie line, PV with ESS and ESS respectively. $P_{I}^{T R}, P_{I}^{P R}$ and $P_{I}^{E R}$ are the remaining generation capacity after demand transferring of node $j$.

Generally, the whole framework of the proposed heuristic algorithm is shown in Fig.3.

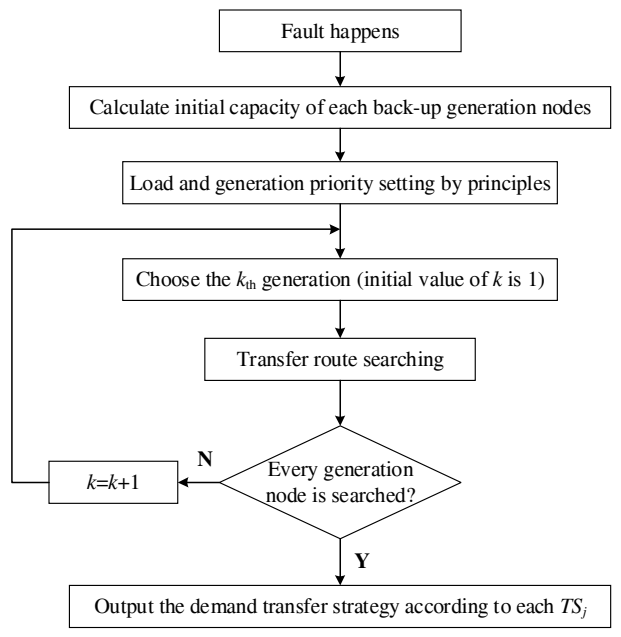

Fig.3 Framework of proposed heuristic algorithm
The essence of the method is similar to the greedy algorithm. During the route searching procedure, the algorithm is making the optimal decision in every steps. As a consequence, it is highly possible that the final demand transfer strategy in only a locally optimal solution. However, by using the proposed method, a practical solution can be achieved rapidly. If the number of feeder nodes is $a$, the number of generation nodes is $b$, the number of transfer time intervals is $c$, the time complexity of the algorithm is $O$ $(a \cdot b \cdot c)$.

\section{CASE Study Simulation}

\section{A. Distribution Network Case Study}

The feeder 3 and feeder 4 in the IEEE RBTS-bus3 distribution network is used to validate the proposed approach. The network structure is shown in Fig.4 and the basic parameters of load, line length, fault rate and fault repairing time can be obtained in ref. [12]. Besides, 8760 hourly load curve [13] and solar data [14] in Topeka, Kansas, US are complemented for the simulation. The three core unarmored copper conductors cable is chosen for the distribution feeder and the tie line [15], whose continuous current carrying capacity is $500 \mathrm{~A}$. The voltage drop limitation is set as 0.95 p.u. The time interval is set as 1 hour.

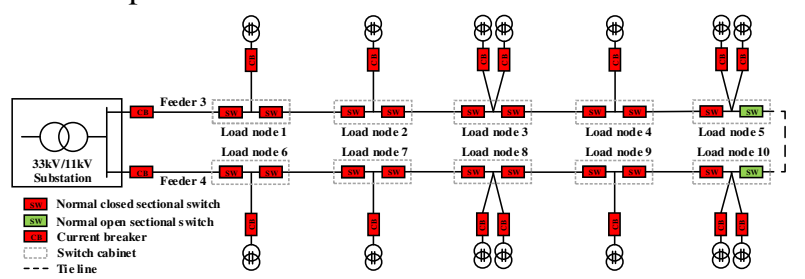

Fig.4 Network structure of case distribution feeder

\section{B. Analysis of Outage Rick Evaluation Indices}

The 8760 hourly load data is clustered into 16 typical load days using the Matlab Neural Network Clustering Toolbox. The largest typical load day is considered as typical peak load day, which occupies 7 days in the year. The load curve of feeder 3 is shown in Fig.1. In order to show the differences between the annual average situation and the worst fault case, the conventional reliability assessment indices ENS, CMI and the outage risk assessment indices are both calculated. The ENS and CMI are evaluated by the Monte Carlo simulation methods. Furthermore, the effects of different alternative planning scheme and operational strategy on the indices are analyzed on the basis of following three cases.

- Case1: Basic case

- Case2: PV with ESS is installed in node 3 and node 8, rated power of $\mathrm{PV}$ is $500 \mathrm{~kW}$ each, maximum power output of ESS is 2MW, Capacity of ESS is 4MWh. ESS is operated with the purpose of reducing network power loss.

- Case3: PV with ESS is installed as the same condition as case 2, ESS keeps its SOC at the highest level when fault happens (14:00) in peak load days.

Comparison of reliability and risk assessment indices in the three cases is illustrate in Tab. II. 
TABLE.II COMPARISON OF INDICES IN DIFFERENT TEST CASES

\begin{tabular}{|c|c|c|c|}
\hline Index & Case1 & Case2 & Case3 \\
\hline ENS(kWh/a) & 5492.13 & 4902.74 & 4881.17 \\
\hline CMI(min/a) & 32.59 & 28.02 & 27.61 \\
\hline RENS(kWh) & 41482.30 & 26291.24 & 10602.81 \\
\hline RCMI(min) & 212.83 & 82.06 & 72.05 \\
\hline RSCI & 1906 & 1906 & 1906 \\
\hline RLCI & 1196 & 466 & 260 \\
\hline
\end{tabular}

Tab. II indicates that although the conventional reliability indices only have a little change in the three cases, the improvement of outage risk indices is much more obvious. These indices can be added into the planning or operation optimization problem to have a more accurate assessment of the candidates.

\section{B. Analysis of Transition Optimization Strategy}

In order to validate the significance of considering the interaction of multiple time intervals during the demand transfer process, a comparison between the proposed algorithm and the algorithm in Ref. [10] is implemented based on the worst case fault in Feeder 3. Ref. [10] optimized the transfer strategy in each time interval independently. The network condition is set as case 3 in the former section. The comparison of transfer process is illustrated in Fig. 4 and the outage risk indices are compared in Table III.

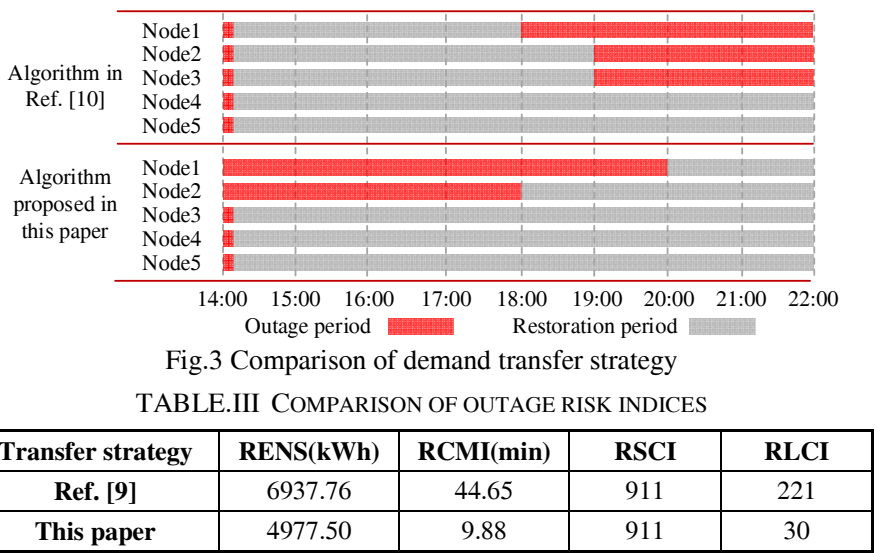

There is no big difference of RENS index in the two strategies because both algorithms are trying to use as much restoration resource as possible. However, fig. 3 shows that the customers in load node 3, 4 and 5 have to be interrupted twice in the strategy of Ref. [10], which cannot be allowed in real distribution network operation in many countries. The reason is that kind of algorithms did not consider if the transfer strategy can continue the restoration in the following periods after it started. That's also why the RLCI index shows a much better result when using the proposed algorithm. Additionally, by programming the proposed algorithm on the Matlab 2013a software on a core i7, 4GB laptop, it only took less than 0.01 seconds to get the transfer strategy result. The calculation efficiency can satisfy the requirement of online applications.

\section{CONCLUSIONS AND FUTURE RESEARCH}

In order to emphasize the outage risk in peak load periods in distribution network, two main work is carried out in this paper. First, four system outage risk evaluation indices are presented on the purpose of assessing the severe interruption impacts of the worst fault case. Second, a multi time interval based demand transfer strategy optimization approach is presented considering the continuity of the restoration procedure. The approach can provide a practical strategy in fault conditions rapidly.

Both the evaluation and the reduction of system outage risk can be effective methods for further research especially in the distribution network planning and operation optimization field.

\section{REFERENCES}

[1] Safdarian A, Degefa M Z, Lehtonen M, et al. "Distribution network reliability improvements in presence of demand response", IET Generation, Transmission \& Distribution, vol. 8, no. 12, pp. 20272035, 2014.

[2] T.a Committee D, "1366 IEEE guide for electric power distribution reliability indices”, The IEEE, Inc.; 2004.

[3] Junyong LIU, Hongjun GAO, Zhao MA, et al, "Review and prospect of active distribution system planning", Journal of Modern Power Systems and Clean Energy, vol. 3, no. 4, pp. 457-467, 2015.

[4] Munoz-Delgado G, Contreras J, Arroyo J M, "Multistage Generation and Network Expansion Planning in Distribution Systems Considering Uncertainty and Reliability", IEEE Transactions on Power Systems, vol.31, no. 5, pp. 3715 - 3728, 2015.

[5] Perrier N, Agard B, Baptiste P, et al, "A survey of models and algorithms for emergency response logistics in electric distribution systems. Part I: Reliability planning with fault considerations", Computers \& Operations Research, vol. 40, no. 7, pp. 1895-1906, 2013.

[6] Abbasi F, Hosseini S M, "Optimal DG allocation and sizing in presence of storage systems considering network configuration effects in distribution systems", IET Generation Transmission \& Distribution, vol.10, no. 3, pp. 617-624, 2015.

[7] Asrari A, Lotfifard S, "Payam M S. Pareto dominance-based multiobjective optimization method for distribution network reconfiguration", IEEE Transactions on Smart Grid, vol. 7, no. 3, pp. 1401-1410, 2016.

[8] Dorostkar-Ghamsari M R, Fotuhi-Firuzabad M, Lehtonen M, et al, "Value of Distribution Network Reconfiguration in Presence of Renewable Energy Resources", IEEE Transactions on Power Systems, vol. 31, no. 3, pp. 1879-1888, 2016.

[9] Sultana B, Mustafa M W, Sultana U, et al, "Review on reliability improvement and power loss reduction in distribution system via network reconfiguration", Renewable \& Sustainable Energy Reviews, vol.66, pp. 297-310, 2016.

[10] Huang X, Taylor G, "Multi-period service restoration of distribution systems incorporating interruptible load", International Transactions on Electrical Energy Systems, vol. 25, no. 9, pp. 1788-1803, 2015.

[11] Guedes L S M, Lisboa A C, Vieira D A G, et al, "A Multiobjective Heuristic for Reconfiguration of the Electrical Radial Network", IEEE Transactions on Power Delivery, vol. 28, no. 1, pp. 311-319, 2013.

[12] Billinton R, uJonnavithula S, "A test system for teaching overall power system reliability assessment", IEEE Transactions on Power Systems, vol. 11, no. 4, pp. 1670-1676, 1996.

[13] Hourly residential and commercial building load datasets, online available at <https://en.openei.org/community/blog/commercial-andresidential-hourly-load-data-now-available-openei>.

[14] National Solar Radiation Data Base, online available at < http://rredc.nrel.gov/solar/old_data/nsrdb>.

[15] 6-36kV Medium Voltage Underground Power Cables, Nexans Energy Networks, 48 Pages, Mar. 2009. 